\title{
DỨBin
}

Technological University Dublin

ARROW@TU Dublin

2010-05-01

\section{A Web and Mobile System for Environmental Decision Support}

\author{
James Carswell \\ Technological University of Dublin, james.carswell@tudublin.ie \\ Keith Gardiner \\ Technological University of Dublin, keith.gardiner@tudublin.ie \\ Michela Bertolotto \\ University College Dublin
}

See next page for additional authors

Follow this and additional works at: https://arrow.tudublin.ie/dmcbk

Part of the Computer Engineering Commons

\section{Recommended Citation}

Carswell, J., Gardiner, K., Bertolotto, M., Rizzine, R.: A web and mobile system for environmental decision support. Decision Support Systems in Agriculture, Food and the Environment: Trends, Applications and Advances, pages 317-338, published by IGI Global. 2010.

This Book Chapter is brought to you for free and open access by the Digital Media Centre at ARROW@TU Dublin. It has been accepted for inclusion in Books/Book chapters by an authorized administrator of ARROW@TU Dublin. For more information, please contact arrow.admin@tudublin.ie, aisling.coyne@tudublin.ie, gerard.connolly@tudublin.ie.

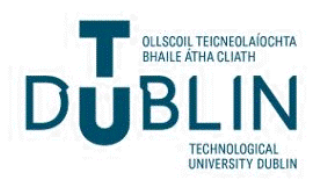




\section{Authors}

James Carswell, Keith Gardiner, Michela Bertolotto, and Andrea Rizzine

This book chapter is available at ARROW@TU Dublin: https://arrow.tudublin.ie/dmcbk/1 


\title{
Digital Media Centre
}

\author{
Articles
}

Dublin Institute of Technology

Year 2009

\section{A Web and Mobile System for Environmental Decision Support}

James D. Carswell

Dublin Institute of Technology, jcarswell@dit.ie

This paper is posted at ARROW@DIT.

http://arrow.dit.ie/dmcart/1 


\section{— Use Licence}

\section{Attribution-NonCommercial-ShareAlike 1.0}

You are free:

- to copy, distribute, display, and perform the work

- to make derivative works

Under the following conditions:

- Attribution.

You must give the original author credit.

- Non-Commercial.

You may not use this work for commercial purposes.

- Share Alike.

If you alter, transform, or build upon this work, you may distribute the resulting work only under a license identical to this one.

For any reuse or distribution, you must make clear to others the license terms of this work. Any of these conditions can be waived if you get permission from the author.

Your fair use and other rights are in no way affected by the above.

This work is licensed under the Creative Commons Attribution-NonCommercialShareAlike License. To view a copy of this license, visit:

- URL (human-readable summary):

http://creativecommons.org/licenses/by-nc-sa/1.0/

- URL (legal code):

http://creativecommons.org/worldwide/uk/translated-license 


\title{
A Web and Mobile System for Environmental Decision Support
}

\author{
${ }^{1}$ James D. Carswell, ${ }^{1}$ Keith Gardiner, ${ }^{2}$ Michela Bertolotto, ${ }^{2}$ Andrea Rizzini \\ ${ }^{1}$ Digital Media Centre, Dublin Institute of Technology, Ireland \\ ${ }^{2}$ School of Computer Science and Informatics, University College Dublin, Ireland
}

\begin{abstract}
Current field data collection methods for many of today's scientific and other observer/monitor type applications are still entrenched in the "clipboard age", requiring manual data transcription to a database management system at some (often much) later date, and only allows for visualisation and analysis of recently captured field data "back in the lab". This chapter is targeted at progressing today's pen \& paper methodology into the spatially enabled mobile computing age of realtime multi-media data input, integration, visualisation, and analysis simultaneously both in the field and the lab. The system described is customized to the specific needs of the Canadian Great Lakes Laboratory for Fisheries and Aquatic Sciences Fish Habitat Management Group requirements for fish species at risk assessment, but is ready for adaptation to other environmental agency applications (e.g. forestry, health-pesticide monitoring, agriculture, etc.). The chapter is ideally suited to all agencies responsible for collecting field data of any type that have not yet moved to a state-of-the-art mobile and wireless data collection, visualisation, and analysis work methodology.
\end{abstract}

Keywords: Spatially Enabled Mobile Computing, Web-Based GIS, Fish Species at Risk, MEMS.

\section{INTRODUCTION}

The latest developments in Internet and mobile technologies have given rise to the implementation of applications easily accessible anytime and anywhere (Hinze et al., 2003; Schiller, 2000; Talukder et al., 2006). Many such applications provide context-based information that can be especially useful for field work, where the manipulation of spatial datasets, including maps of the areas visited, is often required. Examples of professional users that avail of spatial data during their field work are environmental scientists, surveyors, geographers. Spatially enabled computing has the potential to provide crucial decision support to such users by exploiting positional information and associated contextual knowledge. However, notwithstanding the recent advances in spatial and GIS technologies and the availability of increasingly more detailed spatial data, there is still a lack of effective spatial decision support systems.

This chapter describes our experience in the development of a Web-based and Mobile Environmental Management System (MEMS) tailored to deliver context-aware functionality aided by visualization, analysis and manipulation of spatial and attribute datasets. The MEMS datasets are provided by the Canadian Department of Fisheries and Oceans (DFO) and the system is customized to the specific needs of the Great Lakes Laboratory for Fisheries and Aquatic Sciences (GLLFAS) Fish Habitat Management Group requirements for fish species at risk assessment. Before the MEMS system was developed, biologists had only access to the fisheries data from their office. This prevented interacting with the data in a real-time environment, reducing their productivity and effectiveness in the field. Spatially enabling a mobile device allows mobile GLLFAS biologists to make informed decision immediately. 
The functionality required by GLLFAS biologists includes access to geo-referenced maps and imagery, to overlay the current position on a map and to manipulate (e.g. input/edit/query) attribute data in the field while wirelessly connected (where possible) to the office database. Additional functionality also required is the ability to record, edit and view multimedia annotations, perform scientific/common-name conversion and graph generations of results. The traditional "fish species at risk" work-flow, whereby scientists enter textual/pictorial information on paper field data sheets is inefficient, has potential for inaccuracies during both initial recording and subsequent data entry phases, and does not facilitate knowledge-sharing between staff. Also, different types of information may be stored in different locations and valuable time can often be lost trying to correlate data in order to make decisions.

On the contrary, the MEMS system provides many advantages over traditional practice for the GLLFAS scientists. Firstly, it facilitates knowledge-sharing and data analysis/synthesis: indeed, it supports effective communication between different staff at different physical locations (e.g. scientists in the laboratory and colleagues in the field). A standard web-browser is crucial for presenting a familiar "look and feel" interface for staff to input and access information anywhere at any time without having to return to dedicated access points (e.g. in the office).

Furthermore the advanced functionality provided by MEMS allows important multimedia data and associated annotations to be combined with traditional text-based records. Such a system deploys the most recent GIS and mobile technologies and therefore provides a high level of functionality not just for collection and manipulation but also for spatial analysis of the data that was not available to these scientists before. MEMS not only facilitates the work of the GLLFAS biologists but also makes their collected data more reliable and accessible by eliminating both transcription errors and the time elapsed between data collection and data entry, thus saving time as well as tedious paperwork.

MEMS presents important advantages over other existing mobile environmental systems as it provide its users with the possibility of availing of both an on-line version, when network connectivity is available, and an off-line version when network connectivity is lost. The two versions can be automatically and accurately synchronized, therefore providing seamless and continuous use of the application. The system has been deployed on a ruggedized version of a tablet PC. This ensures both sufficient protections for the device during outdoor field work, and a larger display screen for effective input/output.

\section{FUNCTIONAL REQUIREMENTS}

Our research concerned DFO priorities specifically to administer the fish habitat provision of the Fisheries Act, in particular those that are aimed at preventing the harmful alteration, disruption or destruction of fish habitat. This is done to conserve, restore and develop the productive capacity of habitats for recreational, commercial and subsistence fisheries both in the freshwater and marine environments (Minns 1997; Minns 2001).

The traditional workflow of biologists in the DFO includes different phases, i.e. the data collection phase, the data input phase, and the data analysis phase. The collection phase entails recording fish sampling data on paper in the field. Subsequently, at a later stage, the data is inserted manually into the database residing in the office. This process is time consuming and can cause inconsistencies in the data inserted due to human interpretations and typos (e.g. "n.a”, "N/A" or "St.", "Saint”). In addition, the data is usually not validated, thus allowing these errors to enter into the database. During a field trip, biologists obtain samples from fish caught and then record the data collected. For example, they retrieve a previously caught fish from the storage tank on the vessel, identify it, measure its size and other characteristics 
including recording the number caught for that particular species. This inevitability causes the biologist to navigate through many forms, editing some, until all fish are recorded.

In order to identify their requirements, extensive interaction with our targeted end users, namely DFO biologists, took place. This meant participating on DFO field trips and observing their work practices, as well as helping to identify any additional functionality that could facilitate their work. We describe the MEMS functional requirements in this chapter both for a web-based and for a mobile version of the system. The web-based version is used in the office while the mobile version supports scientists in the field.

The functional requirements for the MEMS system include the provision of a real-time, geo-referenced mapping interface that enables the user to view, input and modify field data as well as to annotate maps and photos in a variety of ways. The option to add, delete and query annotations was also required. A multimedia component management module that stores and processes all the multimedia components in the database, was another requirement of the target system.

DFO scientists also need an advanced interface to query the database both through frequently-used static queries and through complex queries created from a series of drop-down menus. More experienced users should also be able to write ad hoc SQL queries. Queries should also be executed, where applicable, based on user location. Biologists also need to process form-based data and map data into different formats for display as well as the ability to transform raw coordinate data from a GPS receiver into a format usable by the application.

Additional functional requirements specific to the mobile version of the system include:

- A Tablet PC or PDA, used as the client; GPRS mobile phone network connection for transmitting data where a wireless connection is possible.

- A GPS receiver connected to the mobile device to determine the current position of the device and deliver it to the application.

- The ability to process data in online and offline modes, depending on network coverage.

- The ability to transmit data over a GPRS network to a central server.

\section{RELATED SYSTEMS}

Several mobile systems have been developed in support of environmental applications. As the main functionality for the DFO biologist in our case relates to collecting data about fish species at risk, existing systems specifically designed to support fishing applications are discussed next.

For example, Terra Vision has developed an Electronic Catch on Effort Reporting System (ECERS) to comply with Australia's regulatory requirements and assist owners and skippers to plan fishing operations. This is achieved by supporting many operations such as data entry/management, data recovery/backup, boarding information and special notification. ECERS offers Vessel Management System (VMS) operations and graphical plot of catch/effort reports. The catch information is stored locally and it is used to create a report, which is then sent to the Fisheries Authorities. Additional ECERS data is also sent to office or home. The software is an end-to-end solution, supporting the vessel client end and also the fishing administrator server end. An OLEDB database is maintained on board the vessel, which can be accessed externally using Microsoft Access, whilst on the land side the system uses Oracle databases to collect the incoming information. ECERS has been developed using Microsoft .Net technology. The connection medium between the central server and the remote client is established using satellite technology (INMARSAT-C). The recorded data is encapsulated in a report, which is sent from and to the client using email communication made secure by encryption. 
OLRAC is a body that specifically provides support for the International fishing industry. Their specialty is the assessment and management of living marine resources. OLRAC developed a software tool called Olfish-2005, which is designed for the electronic collection and management of commercial fishing data. Olfish is a database solution for the capture and management of all fishing data in either real time or from old records. Olfish is capable of collecting, analysing, plotting, mapping, reporting, tracing and transmitting all data related to commercial fishing operations. The software's infrastructure facilitates data sharing from the vessel to the office. Olfish combines a highly sophisticated electronic logbook and GIS system with a fully functional tracing system. Olfish reports comply with the Australian Fisheries Management Authorities (AFAM). These reports are generated automatically and they are sent to the authorities through encrypted email messaging. INMARSAT-C satellites provide the communication channel.

The Secured Harmonized European Electronic Logbook (SHELL) project is a European counterpart to other fisheries management tool such as ECERS and Olfish-2005. This EU funded project aims to open the road for a future European legislation on electronic logbooks for fisheries and is based on previous European research on electronic logbook. This includes developing an operational, cost-effective and secure electronic transfer system that will convey logbook information to and between authority agencies in order to facilitate improved monitoring and control. The deployment of a viable electronic logbook Europe-wide will also improve resource management for scientists as well as the authorities' inspectors. The architecture of the system adopts a monolithic client approach. The On Board System (OBS) computer in the vessel is host to the full SHELL software along with any database required. The communication channel is provided by INMARSAT-C satellites or by wireless or GPRS alternatives. The client hosts the full application. On Shore Authority System (OSAS) hosts the centralized system, which collects data from all the vessels. The deployment of this system is not well documented.

The Trace 2000 software package developed by C-Trace in the UK is essentially an onboard electronic logbook that is being marketed as a traceability tool. While they have not published widely, Trace2000 (T2K) aims at delivering accurate real-time data from fishing vessels to administration companies or operation's offices. The data includes catch data and operational data allowing real-time business decisions to be made. T2K is designed to conform to the EU standards and it also underwent rigorous sea trials in the North Sea. One of the key features of the system is the file-transfer security from and to the fishing vessel. The communication channel varies from INMARSAT-C satellites to Mobile GSM communication. The transmission protocol chosen by the system is the email protocol.

The Norwegian government has developed a logbook system called SatRap. Norway has around 70000 reports registered manually every year and manual handling of all this data is not cost effective. The aim was to meet the fishermen's need for recording and reporting in order to fulfil the requirements of the fisheries authorities. The architecture of this system follows a distributed monolithic approach. The fishing vessel contains a computer hosting SatRap System. The communication channel provided in the fishing vessels is satellite IRIDIUM. These vessels use e-mail for sending reports. The SatRap system provides encryption and authentication mechanisms.

WinCom-C is a system developed by the French Government in order to comply with the national legislation of the fisheries management authorities. WinCom $\mathrm{C}$ was developed by a French company called SODENA which specialise in developing high-technology navigation system software and hardware for PC. The aim of this system is to eliminate paper work by automatically generating catch reports. These catch reports are then sent to the management authorities, while the logbook is automatically sent once the vessel returns to the harbour. The system adopts a distributed monolithic approach. A computer is installed in the fishing vessel hosting the WinCom C software. The computer is then connected to the GPS receiver and other communication means such are INMARSAT-C, MINI-M, and GSM. The systems uses email transmission protocol. 
While the systems presented above each have their advantages and are useful in the specific commercial contexts in which they are applied, none comprises the full set of research requirements for the types of applications needed by Canadian DFO biologists. Furthermore, most were designed for desktop power computers located both onboard ship and in the home office, but not in the hands of a scientist in the field.

An example of system which is closer to our objectives in terms of the application and the technologies used is the Mobile Environment Monitoring System (MEMoS) (Graniero and Miller, 2002) developed at the Multi-purpose Environmental Modelling Facility of the University of Windsor in Canada in collaboration with the Conestoga-Rovers \& Associates (CRA). This system is designed to deliver realtime environmental information using rugged hand-held computers, radio technologies (CRA's OpenRTU), and modular, interoperable software. The system also allows the user to rapidly collect and integrate spatially referenced data from a range of mobile environmental data collectors and update a GIS data warehouse in real-time making data available to a web-based client application instantly. This system adopts a distributed approach and uses a remote base station and mobile data loggers. The remote base station is a vehicle, containing the OpenRTU receiver and a computer with an Oracle Spatial database server. The station allows multiple roving data collectors to wirelessly send recorded structures, where the incoming data are routed to appropriate tables in a local database. The desktop base station contains a web-enabled client application which is used to generate real-time results for the data collected. The mobile data loggers are backpack type equipment containing the OpenRTU transmitter connected to a series of environmental sensors and a rugged Tablet PC for data input. When the base station has finished collecting data, it connects via wired or wireless TCP-IP to the data warehouse, updating the new datasets. The advantage of this system is that it uses an inexpensive communication infrastructure between the base station and the client station. If the communication had to be established via GPRS, running costs could dramatically escalate. A possible disadvantage in running the OpenRTU transmission medium is the actual working range. Also, the disjunctive update of the data warehouse means that information may not be accurate until such updates are completed. Although this system seems to have been successfully applied for their specific case studies, it does not provide many of the functional requirements of our own MEMS system described below.

In general, from this literature review of existing web and mobile spatially enabled systems, we found that many do not contain the required functionality for active field biologists and they are quite difficult to extend if functionality needs adding to existing implementations. In particular, very few systems provide on-line and off-line versions that synchronize effectively. Applications easily deployed onto ruggedized mobile devices are also rare. In our work we have tried to address these issues and provide a suitable ad hoc solution for our selected target user group.

\section{MEMS ARCHITECTURE}

Having identified our user requirements, we then designed a suitable architecture for the MEMS system. This relies on a typical three-tier structure for enterprise information systems, composed of a client layer, an application server layer, and a database layer (Figure 1).

This architecture focuses on the development of services for a versatile, extendible (J2EE) application server, instead of giving GIS capabilities to a large monolithic application. The communication between the client layer and the database are conducted through the application server layer. With this type of architecture, the processing load is more balanced, as each tier of the system can reside on a separate computer (Carswell et al., 2004; Carswell et al., 2008; Rizzini et al., 2006). 
The MEMS system was developed using eSpatial's iSmart Suite application development environment (eSpatial Solutions, 2004). This software is a collection of tools that enables developers to build and deploy spatial applications using sets of standard procedures. It offers developers a high-level development environment which is several times faster than developing the application from base Java. It reduces the need for major software engineering prerequisites that developers typically must have in order to understand and deploy a full spatial application. For the implementation and deployment parts of the system, iSmart Eclipse was used along with iSmart Application Sever.

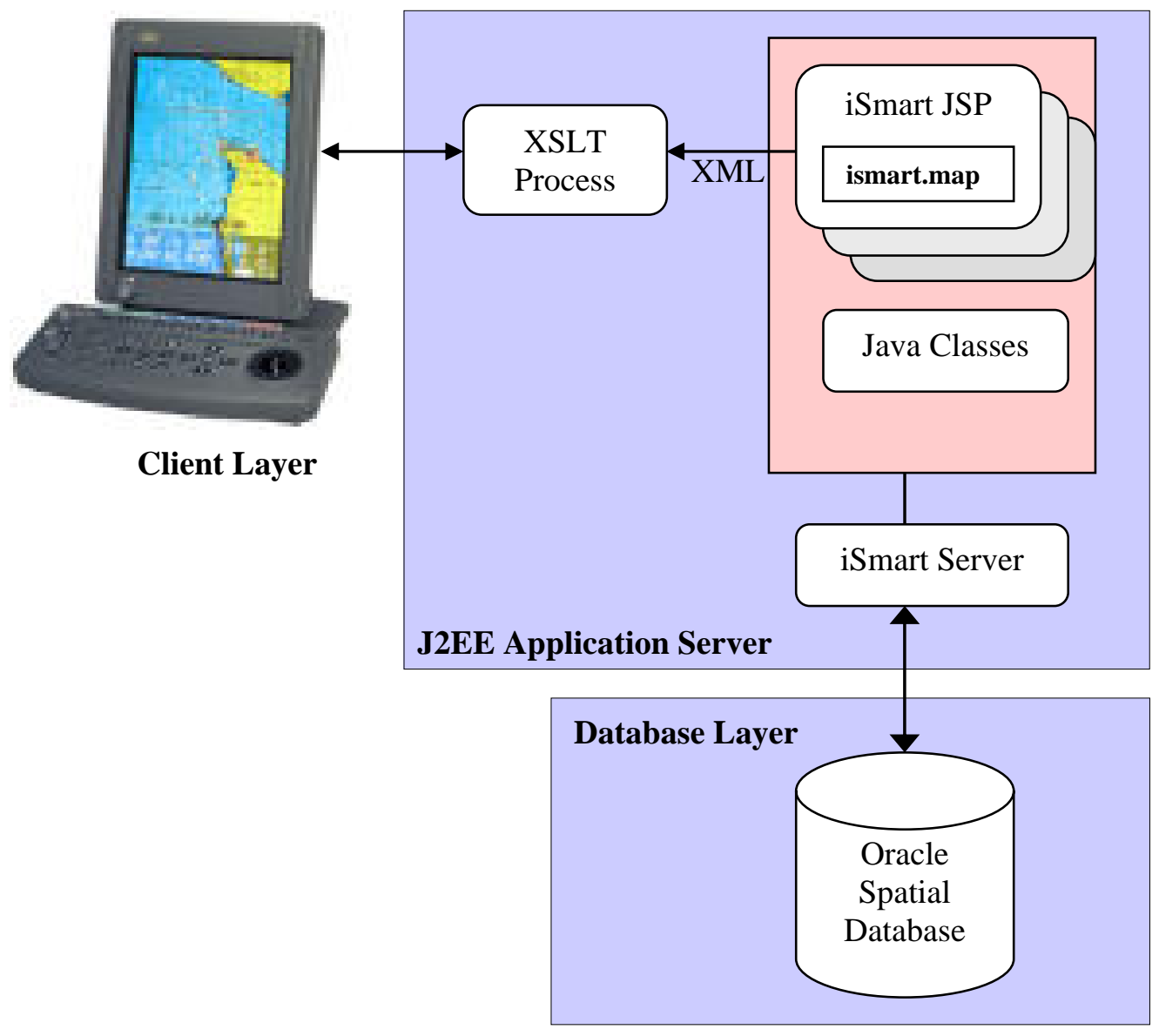

Figure 1. MEMS Architecture

iSmart technology adopts the strategy of focusing the services on the application server layer of the system. This architecture allows for the development of individual components of the system separately, thus maintaining component independence. In this way, different parts of the system can be developed at different stages; some more than others, without affecting the entire system each time a change is made. For example, this architecture has proven ideal for developing Extensible Markup Language (XML)-based applications because all XML/XSLT processing is carried out on the middle tier of the system, without affecting client and/or database tier manipulation or development. In the following we discuss each level of the three-tier architecture, including a brief description of the technologies that are required for each layer.

The client layer of the system consists of a Web-based (i.e. standard web-browser) interface, that enables data-input and analysis functionality directly on the mobile device to provide biologists with the ability to 
input, edit, and annotate data in the field. (Figure 2) As the user navigates within their environment, the position of the user is displayed on a geo-referenced map of the area. This information is used when loading data into the database and for contextually querying the database in real-time. Client hardware consists of a Tablet PC or Personal Digital Assistant (PDA) equipped with a Global Positioning System (GPS) receiver to determine the position of the device. The device also has a General Packet Radio Service (GPRS) network connection for requesting and transmitting data to and from the application server over a wireless phone network. All mobile aspects of the system require a streamlined design, as current speed and bandwidth capabilities of hand-held devices are not yet comparable to those of desktop PCs. Therefore, to deliver content efficiently to these devices it is scaled down using a device-specific content adaptation approach.

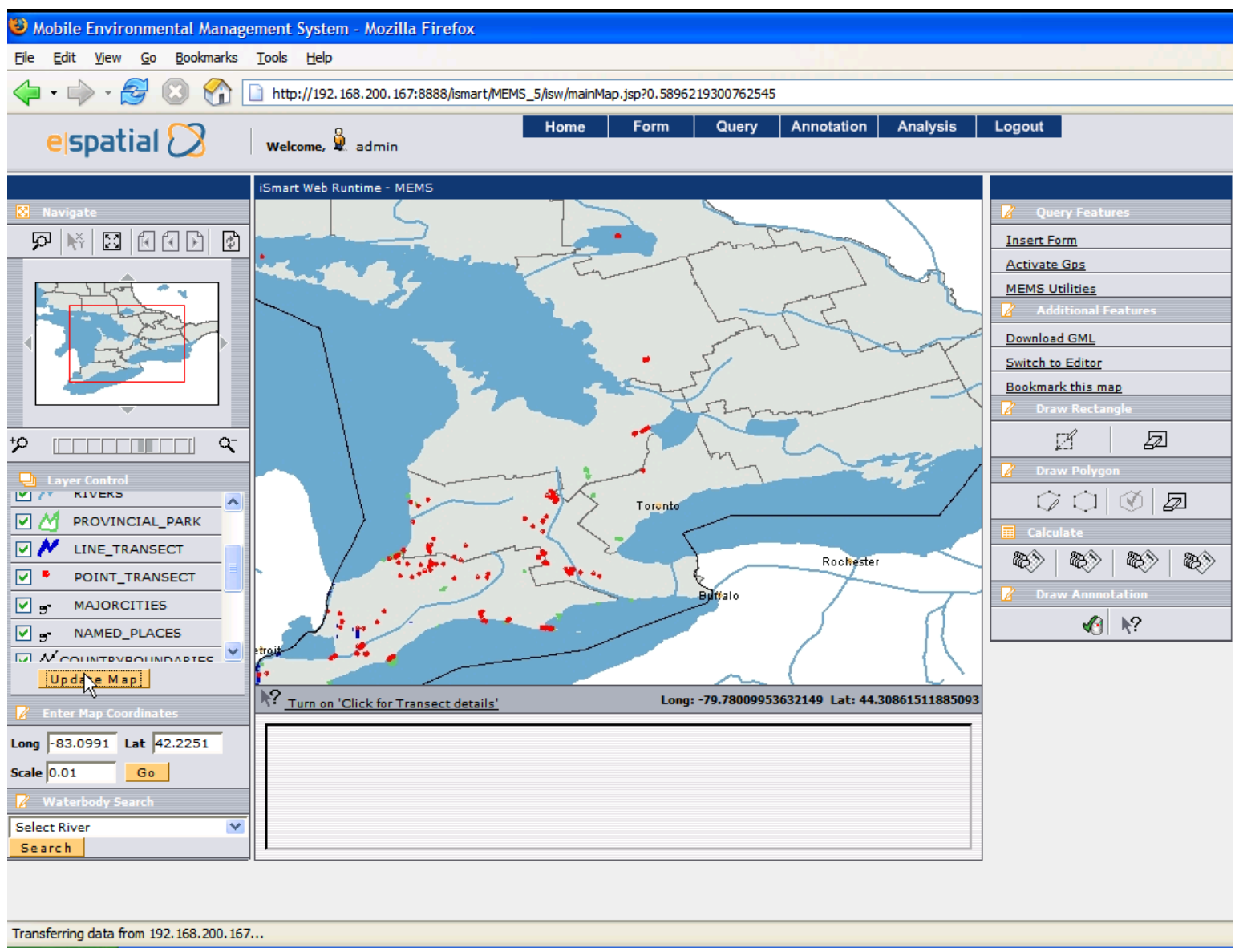

Figure 2. Screenshot of the Graphical User Interface

The application server acts as the main hub between the client and database layers of the MEMS system and consists of a J2EE Application Server made up of two main components - the iSmartWeb Runtime and the iSmart Server. These two components are used to run applications that have been developed using the iSmart Web Designer. (Figure 3) More detail on the sw components (EJBs) developed in the application server layer can be found in (Carswell et al., 2004; Rizzini et al., 2006)

The iSmart Web Runtime is a component integrated with the iSmart Server that executes the web application. When a web page is requested, this component executes the corresponding JSP pages and returns a HTML page back to the client. Within this component, there is a Servlet called the iSMARTWebServlet. This Servlet acts as an interceptor to all incoming HTTP requests and uses control 
logic specified by the developer to decide what actions to take when, for example, a button is pressed on the application web page.

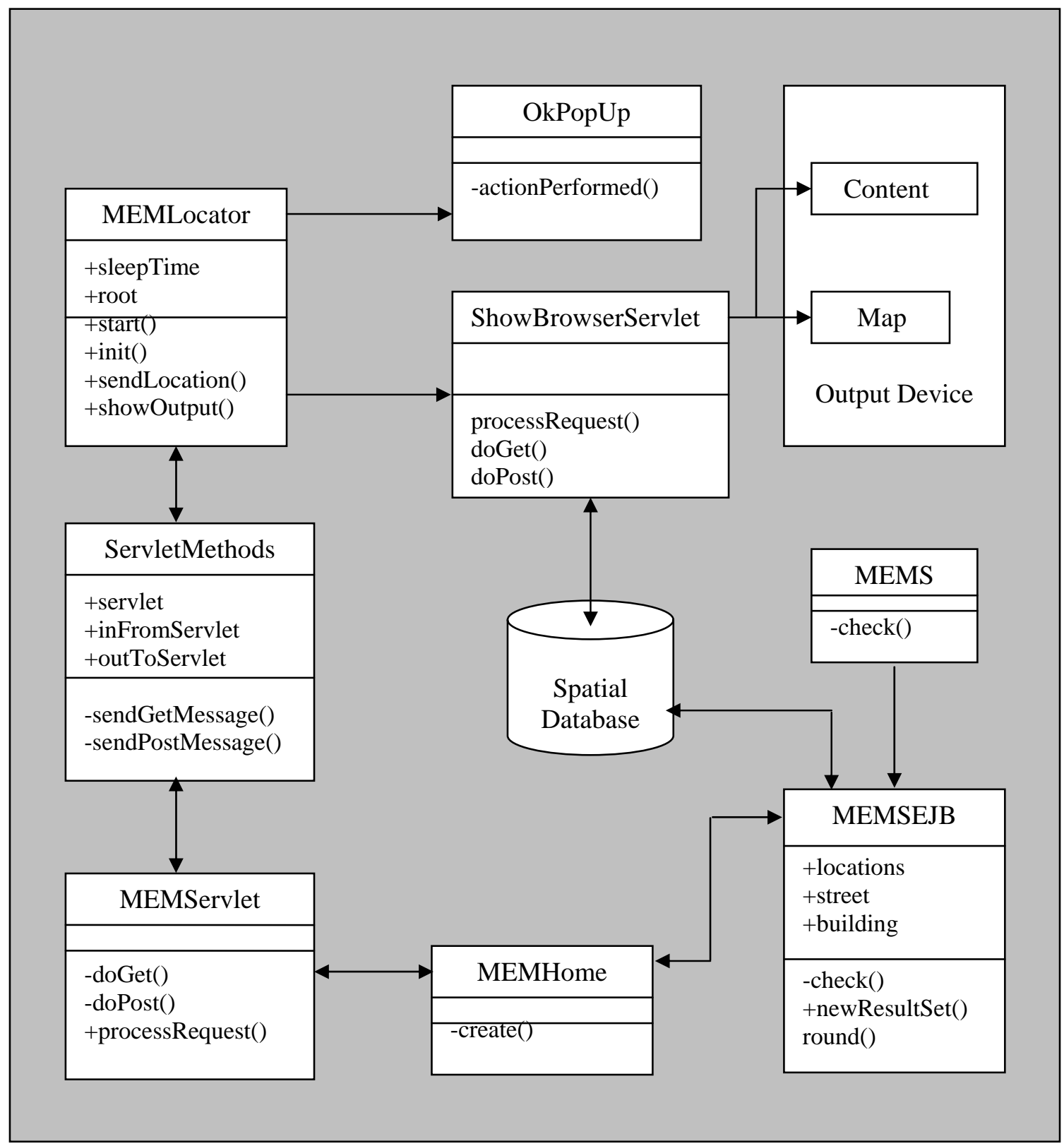

Figure 3. Application Server Layer EJBs and Communications

The iSmart Server contains all the functions and controls in the application server layer and handles all requests made to the application server automatically with the help of an XML file called isw_application.xml. This file is created at deployment time and is used to process requests to the application. This layer of the system is also responsible for determining device-specific capabilities. Using XML and XSLT, the iSmart Server adapts all returned content based on the type of device that makes the initial request. This is achieved by creating an XML file based on the client device request. An existing XSLT stylesheet is then applied to the XML file to create the device specific output. 
The spatial database layer of MEMS is responsible for processing all queries, both spatial and transactional. This tier stores all spatial and non-spatial data together, including raster (map/remote sensing image) data and any metadata as well as the topological properties of these data. It enables Spatial Data Types (SDT) to be inserted, stored, manipulated and queried in the database as they are represented in physical space.

The Oracle Spatial Database Management System was selected as it guarantees interoperability at the data level (Rigaux et al., 2002), conforming to the OGC (Open GeoSpatial Consortium) specifications. Oracle Spatial includes the Spatial Data Option (SDO), a spatial extension to SQL introducing spatial data capabilities, e.g. geocoding and topological queries. Oracle Spatial provides a platform that supports a wide range of applications from automated mapping/facilities management and Geographic Information Systems (GIS), to wireless location services and location-enabled e-business. Oracle Spatial is integrated into the extensible Object Relational Database Management System (ORDBMS), which allows access to the full functionality and security of the underlying DBMS (Lopez, 2003; Sharma 2001). Along with the database, the Oracle proprietary OC4J application server is used. The application server is a component of Oracle, and is installed automatically. The application server acts as deployment platform for Oracle applications.

\section{GLLFAS Data Dictionary}

As mentioned previously, GLLFAS utilized a hardcopy data sheet to record sampling sessions while in the field with the collected data subsequently manually entered into the existing Fish Habitat Management database when back at the office - usually during the winter months. During the migration of the GLLFAS database from MS Access into Oracle, most of the datatypes remained the same except for the start location and stop location. These two fields are now mapped to spatial datatype fields to enable exploitation of the spatial properties inherent with lat/long angular values. Field biologists are also required to sketch a map of the area where the sampling takes place by drawing an outline of the site manually on the data sheet. In MEMS, this process is replaced by utilizing a digital camera to capture an image of the sample-site with self annotation and automatic coordinate input functionality available directly on the image. Further imaging capabilities are exploited when recording fish species captured, kept, and released. In this case MEMS enables the biologist to take a picture of the specimen, look up the scientific name of the fish in the name lookup table and upload all information automatically into the database.

\section{MEMS GRAPHICAL USER INTERFACE AND FUNCTIONALITY}

In this section we describe the implemented system functionality with practical examples of its use through the Graphical User Interface (GUI). The MEMS GUI is divided into three main parts (Figure 2). The left-most panel is the Navigation panel. It contains the functions used to navigate the map (e.g. zoom to area, click x/y coordinates, zoom to extent, back to original view, previous view, refresh map). The navigation map contains a red rectangle which represents the area displayed by the main map. This is designed to help the user navigate more effectively when zoomed into a small area on a map. The Layer Control is located below the navigation panel and is used to select layers to display on the main map. The last two controls on the navigation panel contain Map Coordinates, used to centre the main map with any given coordinates and the Search function control, used for searching previously recorded habitats.

In the centre of the application the Map Panel contains the main map. The main map offers clickable map functionality that enables the user to select features on the map directly and display attribute information about them. The right-most Tools panel contains controls for functions which are explained in the following sections. 


\section{Forms Module}

An important objective of our work is to minimise the amount of tedious data entry work for biologists in the office so as to maximize their hours spent recording new data in the field. To this end, the forms module of the system enables the biologists to view and input data directly while in the field thus reducing the time spent post-processing already recorded field data in the office. Specifically, the forms module enables the biologists to display, insert and edit data using dynamic forms at different stages of the field sampling process. Validation constraints, via drop down lists, were implemented so that incorrect or misspelt data is not inserted into the database. Biologists can also semi-fill forms and revisit them later on in the process to make changes. Figure 4 shows the Data Display Form as an example.

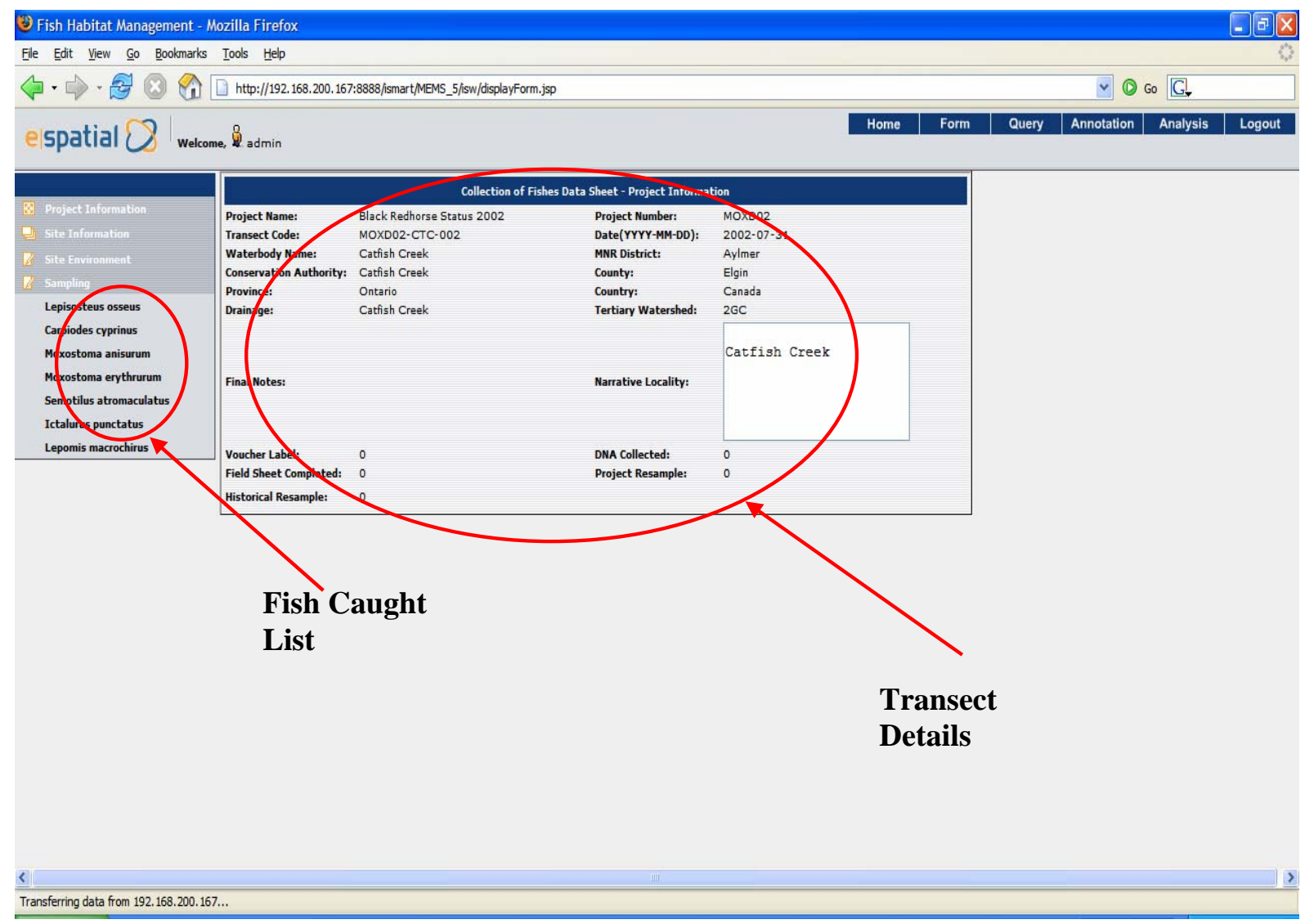

Figure 4. Forms module screen shot

\section{Utilities Module}

This module hosts the following functionality.

- Name Lookup: biologists can identify different types of fish species with their common name (e.g. northern pike), but they are required to record their scientific name (e.g. esox lucius) in the form. In order to help the biologist remember the scientific names of the 269 different fish inhabiting GLLFAS waters, this utility provides a quick translation mechanism. The biologist can select the common name from a drop down menu and instantly obtain the scientific name (Figure 5). 
- Transect Tool: used in order to calculate the distance that a biologist needs to travel in a particular river or water body in order to achieve a satisfactory representative sampling result.

- Accumulation Curve Tool: a graphical generation tool to display the accumulation curve. The accumulation curve is used by biologists to calculate and display how many fish have been caught for a given set of transects and for a particular species (Figure 6a).

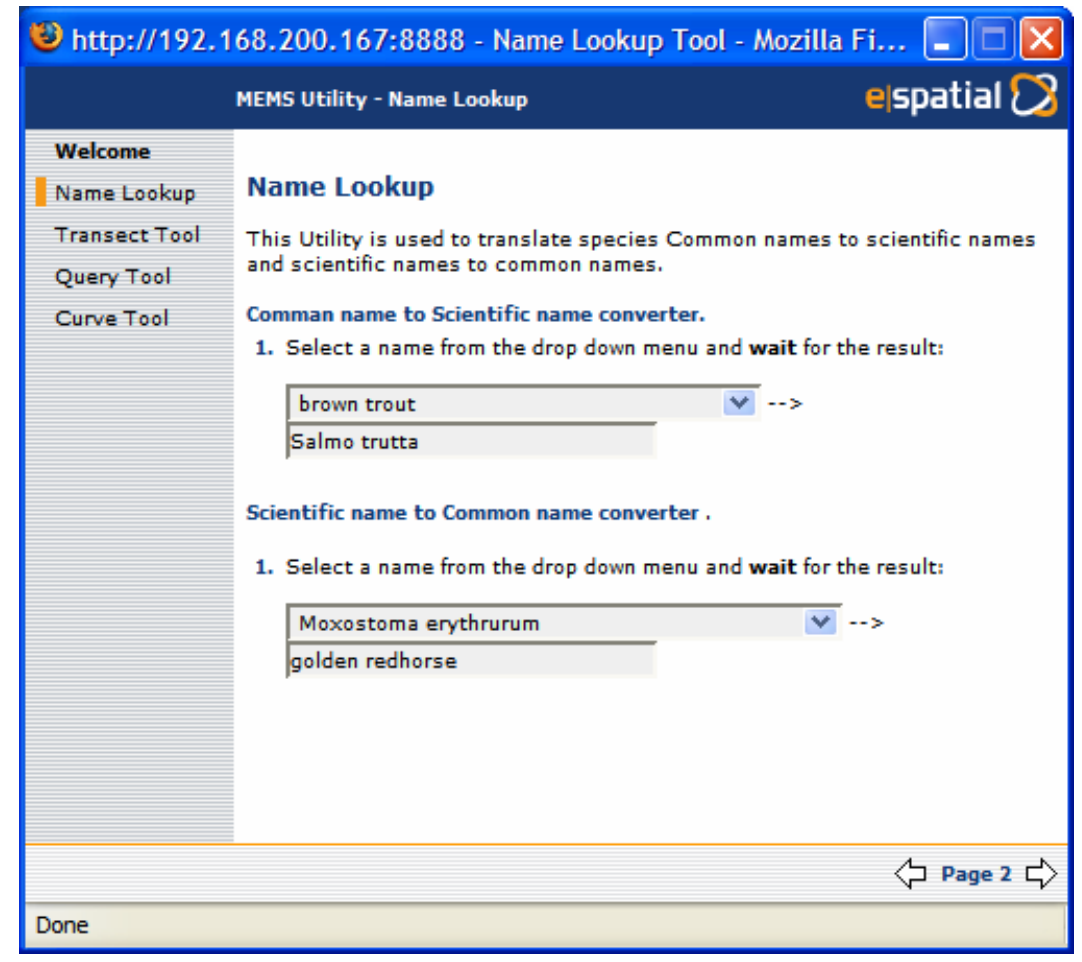

Figure 5.Fish Name Lookup screen

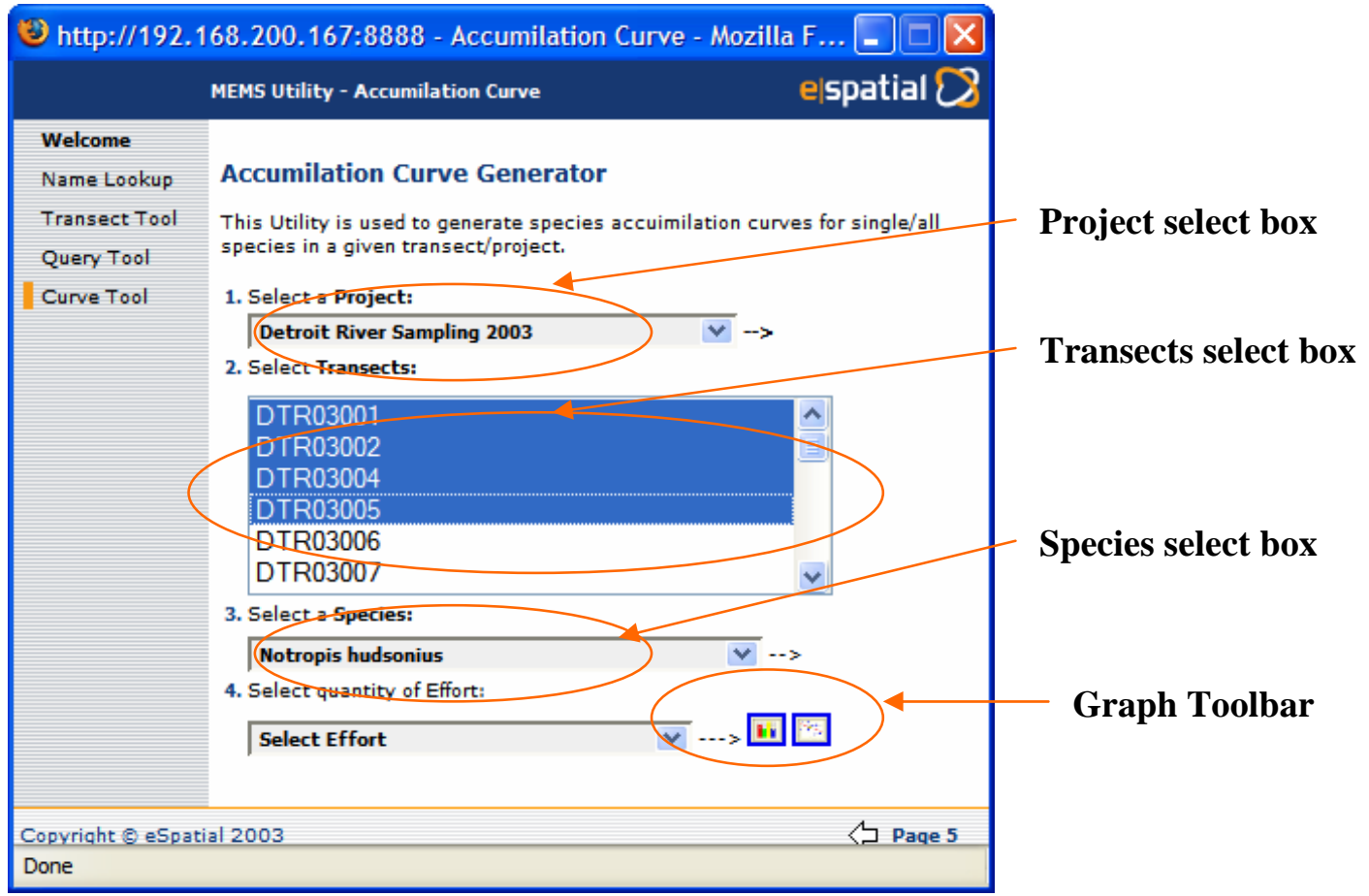


Figure 6a. Accumulation Curve Tool

The Accumulation Curve tool dynamically generates two possible types of graph. The bar chart is displayed by clicking the first button of the "Graph toolbar". There is also a line chart generated by clicking on the second button of the "Graph toolbar". The two types of graph are illustrated in Figure $6 \mathrm{~b}$.
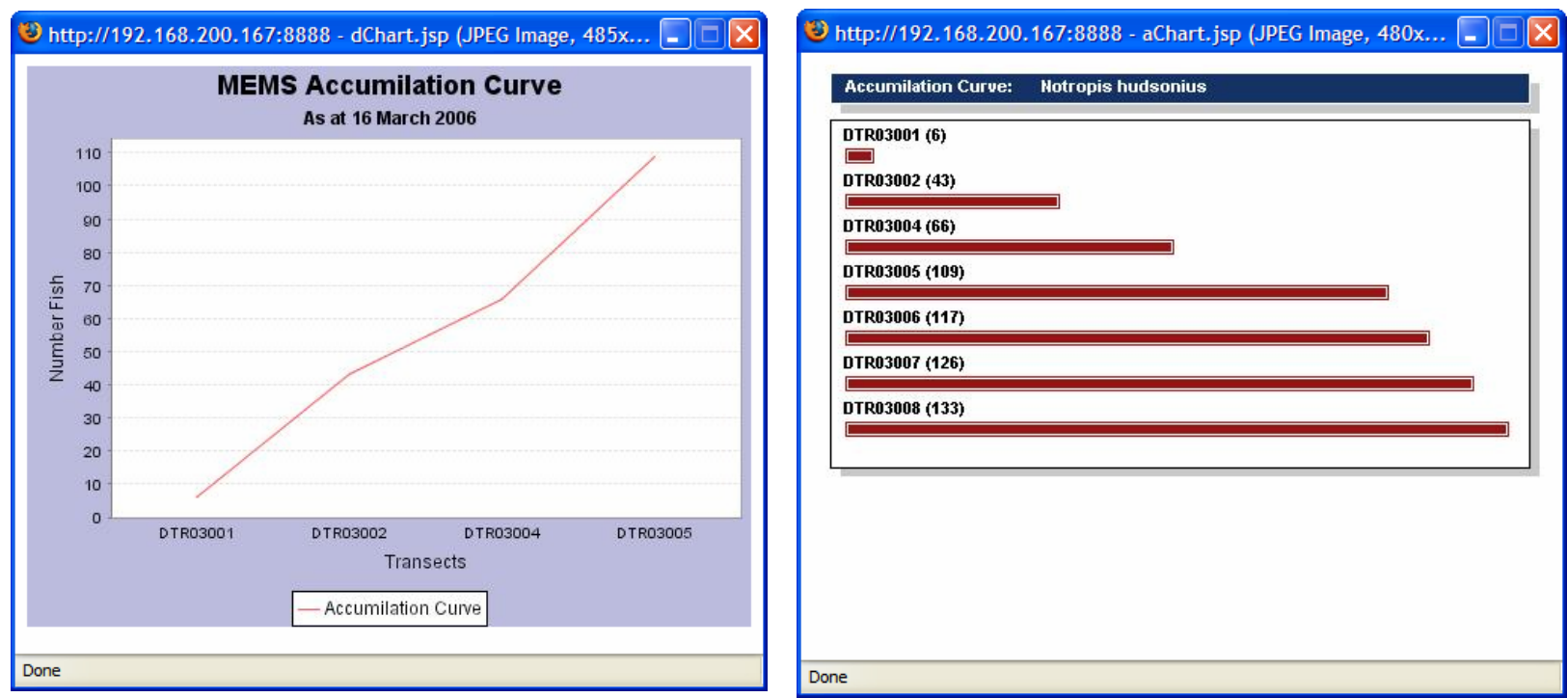

Figure 6b. Accumulation Curve Graphs Generated

\section{Spatial Queries}

The Spatial Queries Tool is an advanced querying tool which enables biologists to quickly and easily query the database without requiring them to have any understanding of Oracle Spatial or the Sequential Query Language (SQL). Users draw a polygonal area on the map where it is then possible to execute a number of predefined spatial queries within the selected area. The polygon highlighted on the map is the actual spatial component and the highlighted buttons hold the predefined queries requested by the GLLFAS biologists. The procedure starts by drawing a polygon or a rectangle query "window" on the map. The dots on the map represent locations where the biologists have previously recorded fisheries data. The result of any given query is parsed and displayed in a pop-up window (Figure 7).

Spatial queries are a particular type of query that use location as their primary search filter. In the MEMS system, Oracle stores non-spatial data relating to a spatial object such as the MNRDistrict and Province of the spatial location (e.g. spawning sites). Although the application does offer advanced non-spatial query functionality, spatial queries can often give a clearer and more simplistic answer to question. Spatial queries are executed by executing Oracle SQL queries against the database and the result is returned as a hybrid ResultSet Java object called GeoResultSet. The GeoResultSet is an iSmart implementation of the ResultSet, which is capable of holding both the spatial and non-spatial attribute ResultSets. The iSmart application can subsequently extract the information from the ResultSet and display it in a simple visual format. In the MEMS prototype, there are three main spatial queries possible. The Range or Buffer query, the Locate on Map query and the Clickable Map query. In this section a brief description of each is given.

\section{Clickable Map Query}

The Clickable Map is an extension of the normal mapping interface, where the particular layer to be queried needs to be specified. The purpose of this query is to enable the DFO biologists in the field to click on objects on the map interface directly and extract information about the object and display it on the 
device. This helps the biologists to get familiar with the surrounding environment and quickly target areas of interest. The spatial query is implemented using JavaScript, JSTL (JSP Standard Tag Library), and the iSmartWeb. The map, which is displayed in the web application, is a JPEG plain image and is refreshed with updated query results each time the user request additional information.

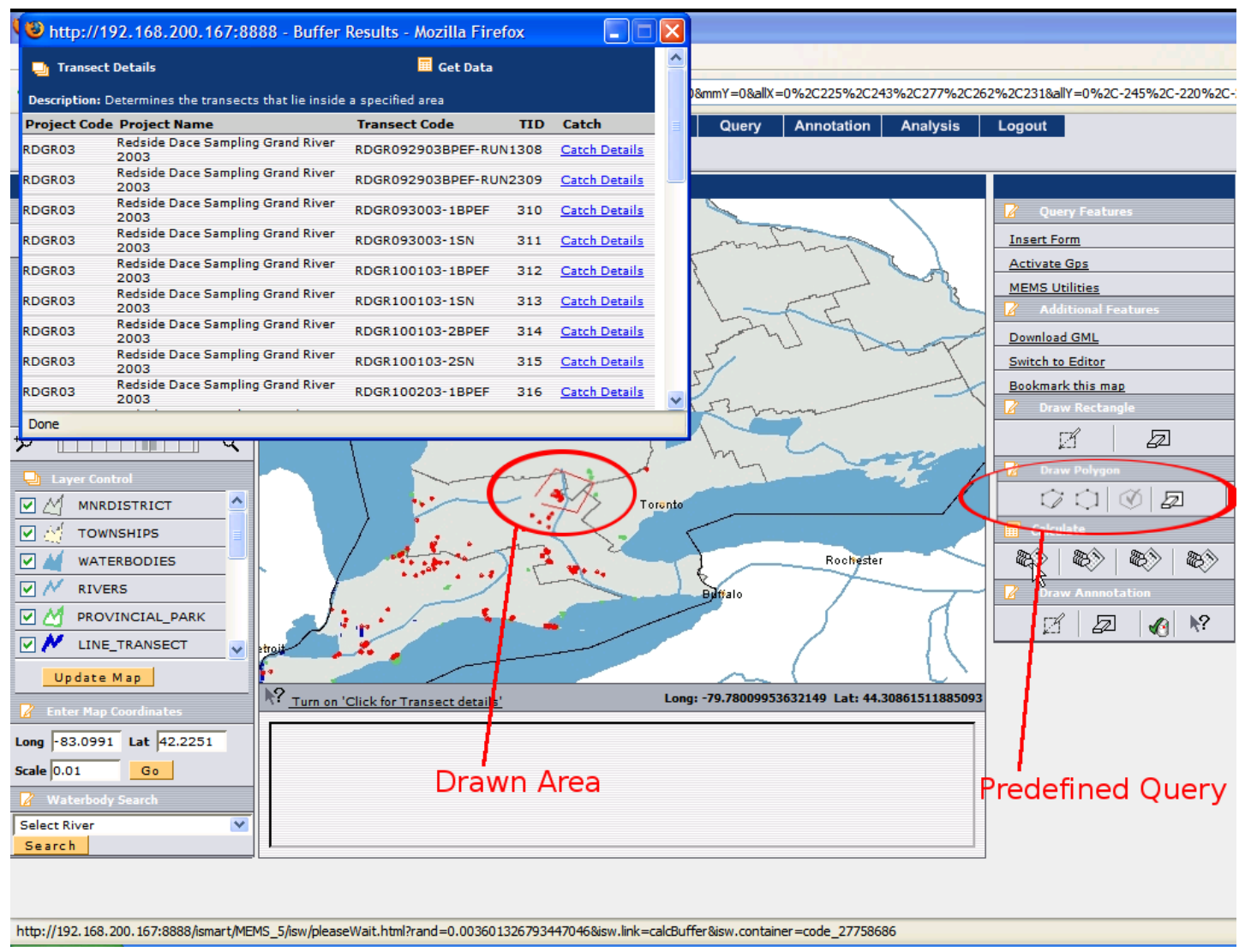

Figure 7. Spatial Queries Tool

\section{Locate on Map Query}

The Locate on Map query consists of two parts and enables the user to locate and display a specific transect on the map. The first part of the query is a text-based search where the user searches for a specific water body. The results are then displayed with a link to the location of the water body on the map and a link which displays more information about the transect. The hyperlinks are linked to iSmart actions, which carry out a query when selected. The locate action is the second part of the query and the result of this query centres the Viewport on the selected transect. This query is particularly useful to biologists in the field when looking for a particular site in a water body. In this way, biologists can locate a transect, and from analysing other details about the position, determine if a sample session is required for that area or not.

\section{Range Query}

Up to this point, the types of queries explained are not technically spatial queries by definition. They are presented as spatial queries because they interact with the application's spatial component but in practice the query itself does not actually interrogate the spatial (lat/long) component. The Range Query is a common truly spatial query and is extremely useful that enables biologists to interrogate the database and gather information about sites within a given distance (range). Once the Clickable Map is activated the 
user is given the option to search the surrounding area for sites. The user specifies a distance in meters from their current location and the query is performed. The result returned is a list of sites that are included within this defined distance.

\section{Drawn (polygon) Query}

This type of query is required when wanting to quickly select a custom area of interest and apply a series of predefined queries. The selection of the area is achieved by drawing the area in the Map Interface. Once the area of interest is created a series of "Spatial queries" can be executed against it. The results will be parsed and displayed in appropriate windows within the web application. This module offers a significant advantage over the other query modules: the ability to effectively draw exactly the area in which the user wants the query to be performed. (Figure 8)

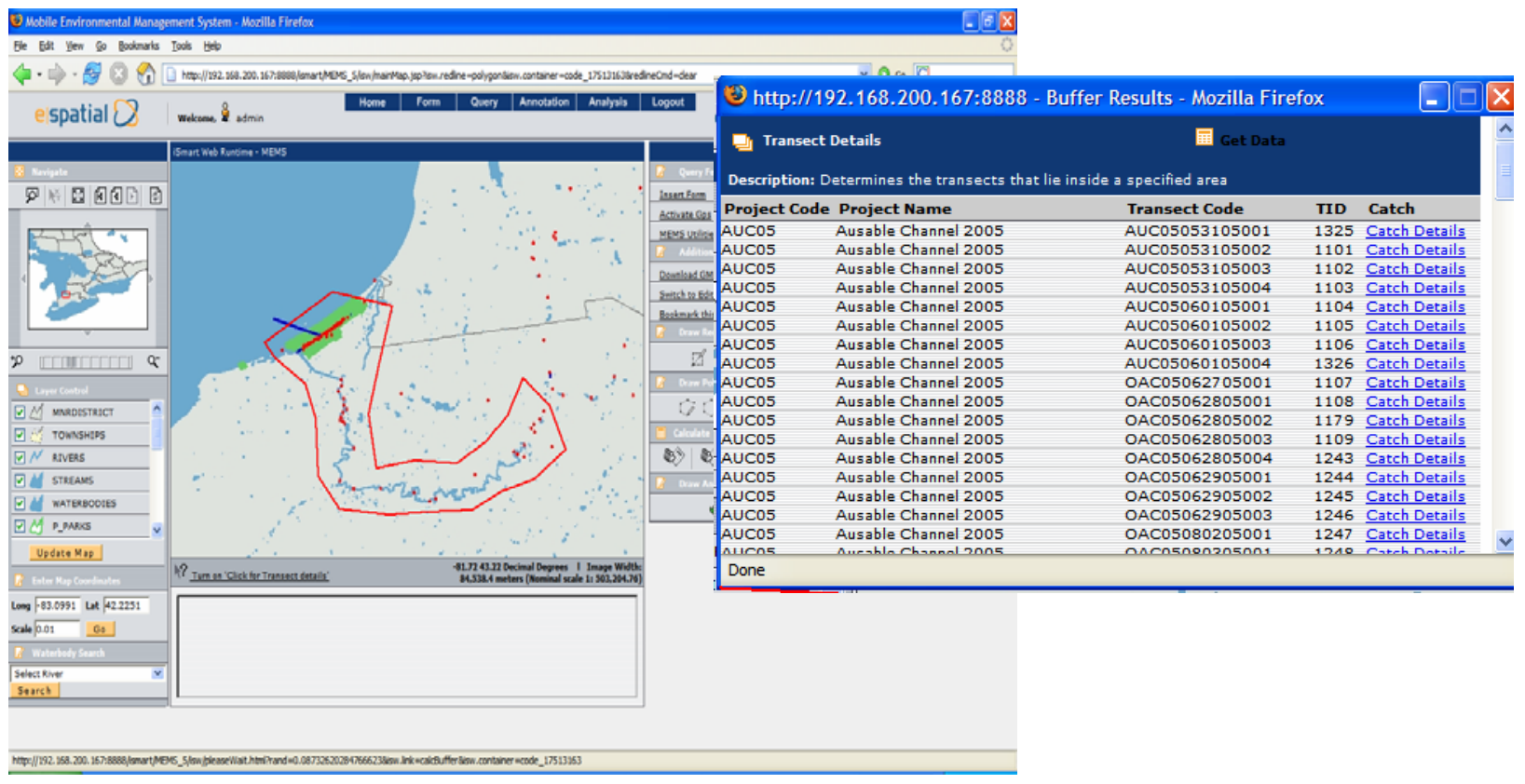

Figure 8. Drawn Query and corresponding pop-up results

This module is instantiated by selecting the drawing tool which corresponds to the first button on the left of the "Drawing Toolbar". The drawing tool uses a complex JavaScript in order to draw and record the points which constitute the polygon. Once the user is satisfied with the polygon, s/he must press the accept button (third button from the left) on the "Drawing Toolbar". The accept button is used to store the geometry of the polygon in the user session. The process starts by reading the points collected in the JavaScript and passing them to a server side component which creates an Oracle Geometry Object called "JGeometry".

In order to execute the query independently of any knowledge of SQL programming, a series of common spatial queries required by the GLLFAS where statically embedded in the application. These queries are shown in the "Query Toolbar" in Figure 9. One example of the query is to return the "Project Name", "Transect Code" and "TID" for all the "Transects" in the drawn polygon. A popup window contains the results of the query and for each entry in the form there are links to generate further queries for more detail information. 


\section{GPS MODULE}

This is a core module as the distributed MEMS application forces the client device to acquire GPS coordinates and then send them to the application server. As the client was not designed to be installation independent it is necessary to execute the Java application remotely.

One of the technologies that Java offers is JNLP (Java Network Launching Protocol). JNLP applications are launched using Java Web Start as part of the Java Runtime Environment. The corresponding GPS application registers each client IP address and host-name with the application server so that multiple feeds can be displayed on the map. This module enables the user to record location data and store it as a spatial component in the database. Once activated the GPS module opens a "GPS Toolbar" and a "GPS Application". This Java application is used to start and stop the GPS receiver. When the GPS receiver has locked onto a steady signal the "GPS Application" displays the current "Latitude" and "Longitude" position, a green light indicating the status and also the signal strength which is the accuracy of the position in meters. Subsequently, the current location is displayed on the map interface and is symbolized by an arrow pointing in the direction the user is moving and a trail showing where the user has been. On the tool bar the user has two buttons, to start and stop recording locations. These buttons are used to save the start and stop position of a transect and the start and stop time for the duration of a particular sampling session - all of which are automatically inserted into the database for each transect.

\section{MULTIMEDIA STORAGE AND ANNOTATION}

The possibility to store, link and annotate multimedia data is another advanced feature of MEMS that allows data to be recorded and inserted into the database on-the-fly. For example, a new species of fish or habitat condition could be encountered and visual evidence would be of great assistance. This functionality enables the user to embed video, audio, text and image annotations on the map. These annotations are uploaded to the database as BLOB data along with their associated coordinates (Figure 9).

Using JSP and Servlet technology, the user is required to enter some text describing the annotation. If the annotations are video, audio or image annotations a file is also required to be uploaded. When this procedure is completed the map is refreshed and an icon representing the annotation is displayed. The user can view the annotations by clicking on the corresponding icon. This action opens a popup window that displays any text associated with the annotation and a link to any multimedia data.

\section{OFFLINE MODE}

The offline module was only considered after our first field visit to the DFO. It was observed that some areas the biologists sample in had only intermittent cellular signal, if at all. As it was difficult to detect or predict network availability before heading to the field, the offline module was designed. The module is implemented using stand-alone Java and developed using the iSmart technology. The Offline Module, also called MEMSOffline, is a standalone application that connects directly and automatically to the online application server back in the office for upload and download of existing and new datasets. For example: before heading to the field and with both tablet PC and office PC linked up, the biologist is asked to select an area of interest for today's sampling (or this week's sampling, etc.) and all data in that area (spatial and attribute) is retrieved and compressed into a zip type file and saved locally on the tablet PC as well as being flagged as "read only" in the database. The MEMSOffline compressed offline file therefore contains all the data required by the field biologist and the application. 


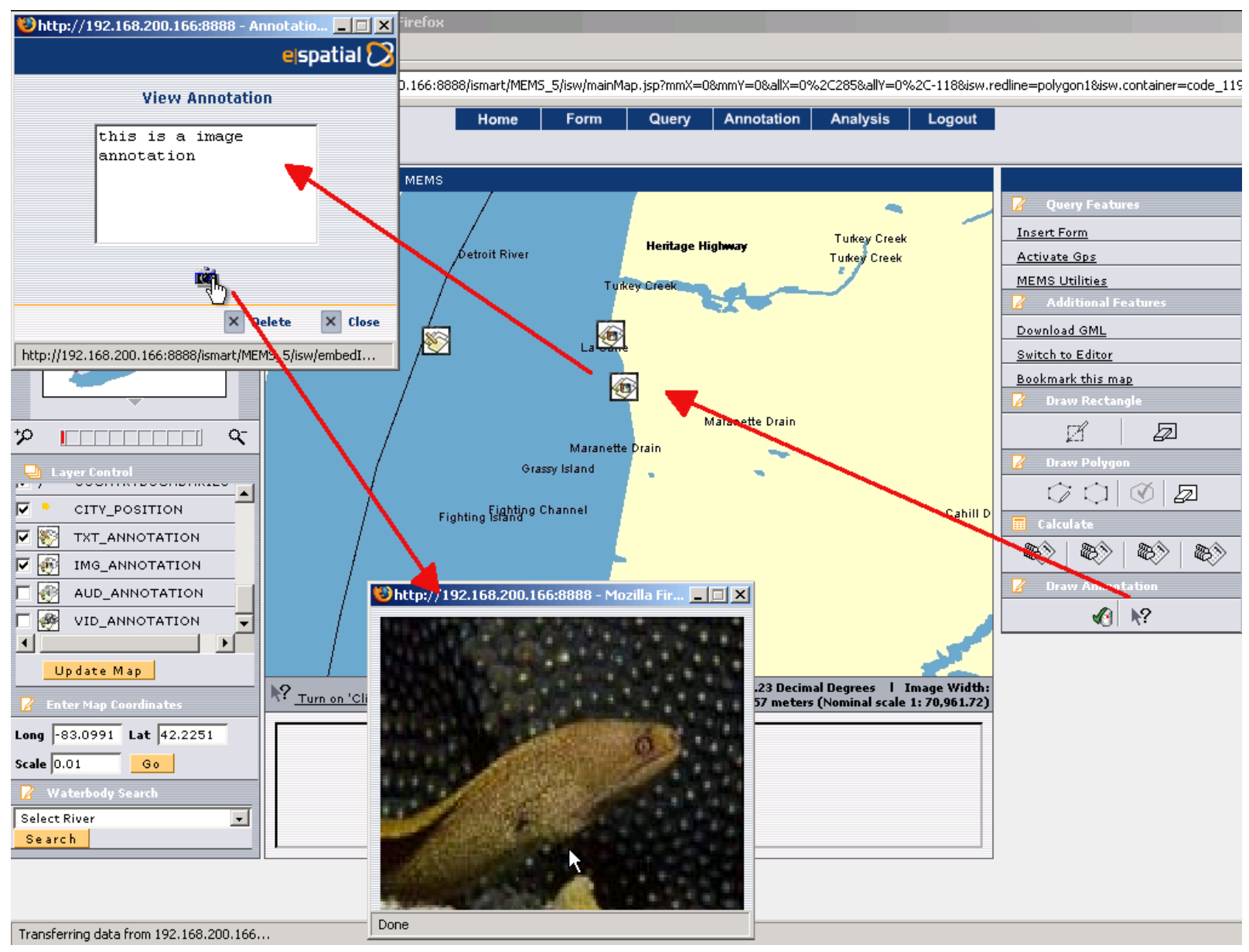

Figure 9. Example of multimedia annotation

This offline application enables the user to work remotely when a GPRS connection is not available. In the field, the offline application reads in the compressed file and displays the map using the iSmart Editor application, enabling the biologists to perform spatial queries, insert new forms, look at previously recorded forms, display GPS feeds and navigate the map as in the case for a live link. It also offers restricted multimedia annotation functionality. Data which is changed or added during the sampling session is stored and saved in the offline file. When the application establishes a network connection and can re-connect to the application server, the data is synchronized with the server and any additions or updates are inserted into the database and the extracted area data is changed back to read/write access.

\section{DISCUSSION AND CONCLUSIONS}

Notwithstanding a widely acknowledged need for more effective spatial support systems to assist realtime decision making for environmental studies, few solutions have so far been provided to address this. Many advances have occurred in fields such as GIS and LBS in the development of spatially enabled webbased and mobile systems. However, these tools are often limited to very specific, often very commercially focused applications. They are not easily extendible and do not take full advantage of the spatial characteristics of the data. They are also often proprietary applications and as such not available for customisation to all. As a consequence, many environmental scientists still use quite rudimentary methods 
for collecting and analysing their field data. Partly this is due to the lack of proper, well-established standards that can be widely adopted in the development of effective low-cost systems. The OGC is investing much effort in this regard but a comprehensive solution is still beyond the horizon. Therefore, timely ad hoc solutions need to be developed to satisfy today's different user requirements.

In this chapter we described our development of a web-based and mobile environmental management system (MEMS) implemented to facilitate the work of biologists in the GLLFAS Fish Habitat Management Group of the Canadian Department of Fisheries and Oceans. As in our case, many environmental applications require field work in areas that do not provide network connectivity. This poses an impediment to the use of mobile systems that can only work in networked environments. Therefore efforts should be devoted to the provision of systems that can adapt to work offline where connectivity is lost and promptly and automatically synchronize with online versions when available.

The MEMS system has been successfully implemented from the technical specifications design. It relies on a Three-Tier Architecture that offers good distribution of processor load, ideal for the development of applications on low-spec devices such as PDAs, and Tablet PCs. Most of the computation is done on the Application Server Layer, and some on the Database Layer, minimizing as much as possible computation on the Client Layer. The result is a lightweight web-browser application with a map interface that enables the user to display fish-sampling transect information based on a water body name, a location, or by clicking on an element on the map. The user is also able to insert new transect information using an intuitive interface.

The system includes an annotation module which enables the users to annotate (via images etc.) points and areas on the maps and also add, edit, delete and query annotations in the database. This functionality has been fully implemented in the web-based application. A GPS module enabling the application to acquire global position has also been fully implemented in the web-based application. In addition, a pure Java standalone offline application was developed to mimic the functionality and the operability of the webbased application. Therefore, the same functionality of GPS module, Form Module, Annotation Module and Synchronization Module, along with all the mapping functionality is also available in the offline version and fully operational on a Tablet PC. Overall, the system offers biologists a spatially-enabled mobile and adaptable service that ensures better utilization of resources. The main benefits provided by the MEMS system include:

- Facilitates knowledge-sharing and data analysis/synthesis.

- Supports effective communication between different staff at different physical locations (e.g. scientists in the laboratory or office, and colleagues in the field).

- Allows important multimedia data and associated annotations to be combined with text-based records.

- Standard web-browser interface allows staff to input and access information anywhere at any time without having to return to dedicated access points (i.e. in the office).

- Reduces errors and paperwork by eliminating time elapsed between data collection and data entry.

However, the advantages of MEMS go beyond the system's functionality to improve both the biologists working environment and in-house data management through automating the vast amounts of fisheries data entry.

MEMS is currently deployed at the GLLFAS headquarters in Burlington Ontario, where extensive field testing has been carried out. Overall response from the users was very favourable once the initial learning curve for a new data collection/entry methodology was overcome. However, this would not be an issue for biologists using the system daily. In terms of cost savings, as the filling out of existing field data sheets was generally mimicked on the MEMS tablet PC (i.e., the same biological measurements need to be 
recorded), its use in the field typically took a similar amount of time for data recording. However, the real cost savings would be given by not having to manually enter the data into the office database after returning from the field. This would save up to 3 months of time every year, depending on the number of sites sampled in the Great Lakes region every summer, and the number of biologists assigned to data entry tasks.

In the future we intend to investigate the adaptability of the MEMS architecture and GUI to different applications and departments within the DFO and elsewhere. We also plan to make the system more adaptable to different user requirements. Much work is being devoted lately to the development of techniques that allow spatial systems to adapt to specific user preferences and tasks. (MacAoidh et al., 2008a; MacAoidh et al., 2008b; Meng et al., 2008) This is a very new research area that aims at assisting users in completing their tasks more efficiently and making more informed decisions. We are currently looking at possible ways to enhance a system such as MEMS with this kind of capability. This involves the application of profiling as well as personalization techniques.

Another area for future work is adding directional query and augmented reality functionality, through the use of digital compass and tilt sensors (accelerometers) that we currently see making their way into mainstream handheld devices. (Rooney et al., 2007; Gardiner et al., 2003; Gardiner et al., 2009) Directional querying is a method by which a device's position and orientation along 2-axis (horizontal and vertical) can be determined with the use of the GPS and compass/accelerometer sensors. This data is then used to build a 2D or 3D "query space”, perhaps confined to the users current field-of-view, in the database to identify what object(s) the device is pointing at - in our case a river bank, weed bed, or spawning ground, etc., and any relevant information (e.g. fish species) associated to it gets returned by the query. The returned information can be communicated back to the user using augmented reality (e.g. visual, auditory or tactile) interfaces.

\section{ACKNOWLEDGEMENTS}

The research presented was funded under the Applied Research Grants scheme of the Enterprise Ireland Innovation Partnership Program (IP-2004-615) and the preparation of this chapter was funded by a Strategic Research Cluster grant (07/SRC/I1168) by Science Foundation Ireland under the National Development Plan. The authors gratefully acknowledge this support.

\section{REFERENCES}

Anliang, N., Fanshun, M., Mingzhu, L. (2008): Mobile Geographical Information Services for 3G Phone; User Wireless Communications, Networking and Mobile Computing; WiCOM '08 4th International Conference on 12-14 Oct. 2008 Page(s):1 - 4

Carswell, J. D., Gardiner K., Bertolotto M., Mandrak N., (2004). Applications of Mobile Computing for Fish Species at Risk Management, Proceedings International Conference on Environmental Informatics of International Society of Environmental Information Sciences (ISEIS2004), Regina, Canada, August 2004.

Carswell, J.D., Gardiner, K.,, Bertolotto, M., Rizzini, A, and Mandrak, N. (2008): A Web-Based and Mobile Environmental Management System; ISEIS Journal of Environmental Informatics 12(1) 9-20 (2008); September 2008.

Doyle, J.; Bertolotto, M.; Wilson, D. (2008): Multimodal Interaction - Improving Usability and Efficiency in a Mobile GIS Context; Advances in Computer-Human Interaction; First International Conference on 10-15 Feb. 2008 Page(s):63 - 68

eSpatial Solutions, (2004): iSmart Web Developers Guide, www.espatial.com. 
Feixiang, C., Chongjun, Y, Wenyang, Y., Xiaoqiu, L., Jianyu Y. (2005): Research on mobile GIS based on LBS; Geoscience and Remote Sensing Symposium; IGARSS '05 Proceedings; IEEE International Volume 2, 25-29 July 2005 Page(s):4 pp.

Gardiner, K., Carswell, J.D. (2003): Viewer-based Directional Querying for Mobile Applications; International Workshop on Web \& Wireless Geographical Information Systems (W2GIS2003); IEEE CS Press; Rome, Italy; December, 2003

Gardiner, K., Yin, J.., Carswell, J.D. (2009): EgoViz - A Mobile Based Spatial Interaction System; $9^{\text {th }}$ International Symposium on Web \& Wireless Geographic Information Systems (W2GIS’09); Springer LNCS; Maynooth Ireland; December, 2009

Graniero, P.A., Miller, H.S. (2002): A mobile environmental monitoring system with real-time database updates; CRESTech Innovation Network Annual Meeting; Toronto ON, 2002.

Hinze, A., Voisard, A., (2003): Locations- and time-based information delivery in tourism; Proceedings 8th International Symposium on Advances in Spatial and Temporal Databases; Santorini Island, Greece; Lecture Notes in Computer Science, Springer-Verlag, pp. 489-507.

Kinal, G. (1998): Inmarsat Services for Navigation; February 1998.

Kotsakis, E. (2002): Secure Information Exchange in Electronic Reporting Systems; Technical Report; 12th June 2002

Kourti, N. (2001): Electronic Logbook for Fisheries: a preliminary system analysis; Technical Report; 24th September 2001

Lemme, P.W., Glenister, S. M., and Miller, A. W. (1999): Iridium aeronautical satellite communications; IEEE Aerospace and Electronics Systems Magazine; vol. 14, no. 11, pp. 11-16, Nov. 1999.

Lopes, X., (2003): Oracle Spatial and Oracle Locator; An Oracle technical white paper; Oracle Corporation, RedwoodCity, CA, December 2003, http://www.oracle.com/technology/products/spatial/pdf/10g_spatial_locator_twp.pdf

Mac Aoidh, E., Bertolotto, M., Wilson, D. (2008a): User Context Analysis from Spatial Interface Interactions, Proceedings 16th European Conference on Information Systems (ECIS 2008), Galway, Ireland, June 9-13, 2008

Mac Aoidh, E., Bertolotto, M., Wilson, D. (2008b): Understanding Geospatial Interests by Visualising Map Interaction Behaviour, Proceedings AGILE Workshop on GeoVisualization of Dynamics, Movement and Change, Girona, Spain, May 2008

Microsoft Corporation, (2001): Universal data access through OLE DB, OLE DB White Papers, http://www.microsoft.com/data/oledb/ , March 2001

Meng, L., Zipf, A. and Winter, S. (eds.), (2008): Map-based Mobile Services -- Design, Interaction and Usability; Springer Series: Lecture Notes in Geoinformation and Cartography. ISBN: 978-3-540-37109-0

Minns, C.K. (1997): Quantifying “No Net Loss” of Productivity of Fish Habitats; Canadian Journal of Fisheries and Aquatic Sciences (Journal Canadien des Sciences Halieutiques et Aquatiques); 54 , pp. 2463-2473. 
Minns, C. K., (2001): Science for Freshwater Fish Habitat Management in Canada: Current Status and Future Prospects; Aquatic Ecosystem Health and Management; 4, pp. 423-436.

OpenRTU eSolutionsGroup: http://www.openrtu.com/

Olrac: http://www.olrac.com/

Rigaux, P., Scholl, M., Voisard, A., (2002): Spatial Databases; Academic Press; Morgan Kaufmann Pub.

Rizzini, A., Gardiner, K., Bertolotto, M., Carswell, J., (2006): MEMS Mobile GIS: A Spatially Enabled Fish Habitat Management System; 6th International Symposium on Web and Wireless Geographical Information Systems), Hong Kong, December 2006, Springer LNCS; pp. 113-122.

SatRap: http://www.fiskeridir.no/fiskeridir/english/resource-management/fmc-norway

Schiller, J., (2000): Mobile Communications; Addison Wesley Publishers.

Sharma, J., (2001): Oracle Spatial; An Oracle technical white paper; Redwood City, CA, May 2001.

Talukder, A., Yavagal, R., (2006): Mobile Computing; McGraw Hill.

TerraVision: http://www.sat.com.au/terravision/

WinCom-C: http://www.sodena.net/UK/specific/wincomc.htm

Open Geospatial Consortium, Inc. http://www.opengeospatial.org/

Rooney, S., Gardiner, K., Carswell, J.D., Kilfeather, E. (2007): An Open Source Approach to Wireless Positioning Techniques; The 5th International Symposium on Mobile Mapping Technology (MMT’07);

The ISPRS International Archives of Photogrammetry, Remote Sensing and Spatial Information Sciences; Padua, Italy; May 28-31, 2007

Yuting, S., Shuliang, Z., Yiming, Z., GuoNian L., (2008): Design and Implementation of Integration Framework for Terminal-Oriented Mobile GIS Data Collection; Grid and Pervasive Computing Workshops, 2008. GPC Workshops '08. The 3rd International Conference on 25-28 May 2008 Page(s):151 - 157 